\title{
Glucagon-like peptide-1 activates endothelial nitric oxide synthase in human umbilical vein endothelial cells
}

\author{
Li DING, Jin ZHANG* \\ Department of Endocrinology, First Affiliated Hospital of China Medical University, Shenyang 110001, China
}

\begin{abstract}
Aim: To investigate the effects of glucagon-like peptide-1 (GLP-1) on endothelial NO synthase (eNOS) in human umbilical vein endothelial cells (HUVECs), and elucidate whether GLP-1 receptor (GLP-1R) and GLP-1(9-36) are involved in these effects. Methods: HUVECs were used. The activity of eNOS was measured with NOS assay kit. Phosphorylated and total eNOS proteins were detected using Western blot analysis. The level of eNOS mRNA was quantified with real-time RT-PCR.

Results: Incubation of HUVECs with GLP-1 (50-5000 pmol/L) for 30 min significantly increased the activity of eNOS. Incubation of HUVECs with GLP-1 (500-5000 pmol/L) for 5 or 10 min increased eNOS phosphorylated at ser-1177. Incubation with GLP-1 ( $5000 \mathrm{pmol} / \mathrm{L})$ for $48 \mathrm{~h}$ elevated the level of eNOS protein, did not affect the level of eNOS mRNA. GLP-1R agonists exenatide and GLP-1(9-36) at the concentration of 5000 pmol/L increased the activity, phosphorylation and protein level of eNOS. GLP-1R antagonist exendin(9-39) or DPP-4 inhibitor sitagliptin, which abolished GLP-1(9-36) formation, at the concentration of 5000 pmol/L partially blocked the effects of GLP-1 on eNOS.

Conclusion: GLP-1 upregulated the activity and protein expression of eNOS in HUVECs through the GLP-1R-dependent and GLP-1(9-36)-related pathways. GLP-1 may prevent or delay the formation of atherosclerosis in diabetes mellitus by improving the function of eNOS.
\end{abstract}

Keywords: glucagon-like peptide-1; human umbilical vein endothelial cell; endothelial NO synthase; atherosclerosis; diabetes mellitus

Acta Pharmacologica Sinica (2012) 33: 75-81; doi: 10.1038/aps.2011.149; published online 28 Nov 2011

\section{Introduction}

Endothelial nitric oxide synthase (eNOS), which synthesizes nitric oxide (NO) from the substrate L-arginine in endothelial cells (ECs), plays an important role in regulating a broad spectrum of functions in the cardiovascular system, including vasorelaxation, the inhibition of leukocyte-endothelial adhesion, vascular smooth muscle cell (SMC) migration and proliferation, and platelet aggregation ${ }^{[1]}$. NO is the most important endothelium-derived vasodilator and has a potent anti-atherosclerotic effect because of its anti-oxidative, anti-inflammatory and anti-coagulatory properties ${ }^{[2,3]}$. Pathological changes such as insulin resistance and the metabolic alterations in type 2 diabetes mellitus (T2DM) can lead to eNOS dysfunction and relatively low NO production, which are now considered the major mechanisms of macrovascular complications in T2DM.

Glucagon-like peptide-1 (GLP-1) is a gut hormone that is

\footnotetext{
* To whom correspondence should be addressed.

E-mail zhangjininsy@gmail.com

Received 2011-05-20 Accepted 2011-09-30
}

released into the bloodstream after feeding. Potentiating the insulinotropic action of glucose, GLP-1 is effective in controlling glucose by stimulating insulin secretion. The impaired secretion of GLP-1 in patients with T2DM has been considered to be one of the mechanisms underlying their abnormal glucose metabolism ${ }^{[4-7]}$. Thus, its analogues have been used clinically to treat $\mathrm{T}_{2} \mathrm{DM}^{[8]}$. Recently, many researchers have turned their attention to the cardiovascular effects of GLP-1 and have found that GLP-1 can induce endothelium-dependent vasorelaxation ${ }^{[9-11]}$ and improve the response of vessels to other vasodilators $^{[12-14]}$. Furthermore, GLP-1 can elevate the NO levels in coronary effluent from mouse hearts, and its vasorelaxing effect can be blocked by an eNOS inhibitor ${ }^{[11]}$, suggesting that GLP-1 upregulates eNOS. Taken together, we reason that GLP-1 may be an endogenous vascular-protective hormone and an extremely effective drug target for pharmacological intervention in T2DM patients.

GLP-1 exerts its actions through GLP-1 receptor (GLP-1R), which is also expressed in the endothelium ${ }^{[11,13,15]}$. Native GLP-1, existing mainly as an intact, biologically active form 
(GLP-1 [7-36 amide]), is rapidly degraded after secretion by depeptidyl peptidase-4 (DPP-4) to its inactive form, N-terminally truncated GLP-1(9-36). GLP-1(9-36) has a weak affinity to GLP-1R and was previously considered to have no biological function. However, some research has suggested that GLP-1(9-36), which offers significant cardioprotection against ischemia-reperfusion injury and induces vasodilation ${ }^{[10,11,16]}$, may affect GLP-1 function, at least in the cardiovascular system.

In the present study, to improve our understanding of the mechanism by which GLP-1 may exert cardiovascularprotective effects, we investigated the effect of GLP-1 on the activation and expression of eNOS in human umbilical vein endothelial cells (HUVECs). In addition, we investigated whether the GLP-1R-dependent or GLP-1(9-36)-related pathways are involved in these effects.

\section{Materials and methods Cell culture}

Primary human umbilical vein endothelial cells (HUVECs) derived from normal human placenta umbilical cord tissues were purchased from Pricells Company (Wuhan, China). They were verified with vWF, FactorVIII and CD31 (P-CAM), and frozen in liquid nitrogen at the end of primary culture. After the purchase, they were recovered and sub-cultured in endothelial cell medium containing 5\% fetal bovine serum (FBS) and 1\% endothelial cell growth supplement (ScienCell, San Diego, CA, USA) in a humidified atmosphere of $95 \%$ air and $5 \% \mathrm{CO}_{2}$ at $37^{\circ} \mathrm{C}$. The cells from the 3rd-6th passages were used in all of the experiments. When grown to 70\%-90\% confluence, the cells were incubated in the presence or absence of GLP-1 (ProSpec, Rehovot, Israel), exenatide (a GLP-1R agonist) (Lilly, Indianapolis, IN, USA), GLP-1(9-36) (Chinese Peptide, Hangzhou, China), exendin(9-39) (a GLP-1R antagonist) (Sigma, St Louis, MO, USA) or sitagliptin (an inhibitor of DPP-4) (HKBY, Beijing, China) for various times and then collected for further analysis.

\section{Measurement of eNOS activity}

The activities of eNOS were assayed with an NOS assay kit (Beyotime, Haimen, China) according to the manufacturer's instructions. The cells were grown to $90 \%$ confluence in 96-well microplates and incubated with or without 5-5000 pmol/L GLP-1, GLP-1(9-36) or exenatide in a $200 \mu \mathrm{L}$ system containing sufficient substrates of NOS (eg, NADPH and L-Arginine) and 3-Amino, 4-aminomethyl-2', $7^{\prime}$-difluorescein, diacetate (DAF-FM DA), a cell-permeable fluorescent substance. After incubation at $37^{\circ} \mathrm{C}$ for $30 \mathrm{~min}$, the fluorescence intensity (FI) was detected using a microplate reader (Infinite ${ }^{\circledR} 200$ Pro, Tecan, Switzerland) with an excitation wavelength of $495 \mathrm{~nm}$ and an emission wavelength of $515 \mathrm{~nm}$. Each plate also included at least 2 negative control wells to which no cells were added. The relative activities (RA) of eNOS were calculated as follows:

$$
R A=\left(F I_{\text {sample }}-F I_{\text {negative }}\right) /\left(F I_{\text {control }}-F I_{\text {negtive }}\right)
$$

\section{Western blot analysis}

Western blot analysis was used to quantify the total and phosphorylated (ser-1177) levels of eNOS proteins and to detect the expression of GLP-1R and DPP-4 in HUVECs. Cells were washed 3 times with ice-cold PBS and then lysed with ice-cold RIPA lysis buffer (Beyotime) supplemented with $1 \mathrm{mmol} / \mathrm{L}$ phenylmethanesulfonyl fluoride (Beyotime) and phosphatase inhibitors (YuanPingHao Bio, Beijing, China). The cell lysates were centrifuged $\left(12000 \times \mathrm{g}, 12 \mathrm{~min}, 4^{\circ} \mathrm{C}\right)$, and the supernatants were collected. Protein concentrations were determined using a BCA kit (Beyotime). Equal amounts of samples $(50 \mu \mathrm{g})$ were denatured and subjected to 10\% SDS-PAGE. The separated proteins were then electro-transferred onto a PVDF membrane blocked with $5 \%(w / v)$ fat-free milk powder in Tris-buffered saline with Tween (TBST) for $2 \mathrm{~h}$. The membranes were incubated overnight at $4{ }^{\circ} \mathrm{C}$ with primary antibodies as follows: anti-eNOS (1:400), anti-phospho-eNOS (ser-1177) (1:200), antiGLP-1R (1:400), anti-DPP-4 (1:400) and anti- $\beta$-actin (1:500) (Santa Cruz Biotechnology, Santa Cruz, CA, USA). The membranes were then washed and incubated with horseradish peroxidase-conjugated goat anti-rabbit IgG (1:5000) in TBST for $2 \mathrm{~h}$ at room temperature. The immunostained proteins were visualized by enhanced chemiluminescence (ECL; Santa Cruz Biotechnology). The intensities of the bands were quantified using a Gel Doc ${ }^{\mathrm{TM}} \mathrm{XR}$ imaging system (Bio-Rad Laboratories, Hercules, CA, USA) and Quantity One software.

\section{Real-time RT-PCR}

HUVECs were lysed on ice with TRIzol ( $1 \mathrm{~mL} /$ well) (GBCBIO, Guangzhou, China), and total RNA was extracted with chloroform and isopropanol according to the manufacturer's protocol. The extracted RNA was quantified using NaNoDrop (NaNoDrop Technologies, Wilmington, DE, USA). cDNA was synthesized from $1 \mu \mathrm{g}$ of total RNA using the PrimeScript ${ }^{\circledR}$ RT Reagent Kit (Takara, Dalian, China). The levels of eNOS transcripts were quantified with real-time RT-PCR using SYBR ${ }^{\circledR}$ Premix Ex Taq ${ }^{\mathrm{TM}}$ (Takara Bio) according to the manufacturer's instructions. The primer pairs used in the real-time PCR for human eNOS were 5'-TCCACGATGGTGACTTTGGCTA-3' (sense) and 5'-GCTGTCTGCATGGACCTGGA-3' (antisense), and the primer pairs used for the human housekeeping gene $\beta$-actin were 5'-TGGCACCCAGCACAATGAA-3' (sense) and 5'-CATAGTCATAGTCCGCCTAGAAGCA-3' (antisense). The PCR reactions were carried out in a Rotor-Gene 6000 system (Corbett Life Science, Australia) under the following conditions: $95^{\circ} \mathrm{C}$ for $30 \mathrm{~s}, 45$ cycles at $95^{\circ} \mathrm{C}$ for $5 \mathrm{~s}$ and $55^{\circ} \mathrm{C}$ for $30 \mathrm{~s}$. The expression level of eNOS relative to $\beta$-actin (the internal reference gene) was calculated and adjusted against the expression levels of untreated samples, which were deemed to be 1.0. The calculation formula is as follows: eNOS relative gene expression $=(\mathrm{eNOS}$ gene concentration in treatment group $/ \beta$-actin gene concentration of the same sample)/(eNOS gene concentration in control group/ $\beta$-actin gene concentration of the same sample). 


\section{Statistical analysis}

A statistical analysis was performed with SPSS version 13.0 (SPSS Inc, Chicago, IL, USA). Data are presented as the mean $\pm S D$. Multiple comparisons were evaluated by a oneway ANOVA followed by the S-N-K test. $P<0.05$ was considered statistically significant.

\section{Results}

\section{GLP-1 promotes eNOS activities in HUVECs}

The incubation of HUVECs with GLP-1 for $30 \mathrm{~min}$ increased eNOS activity (Figure 1A). GLP-1 increased eNOS activity at $5 \mathrm{pmol} / \mathrm{L}$, but the difference was not significant. Higher concentrations of GLP-1 (50-5000 pmol/L) significantly enhanced eNOS activity. As indicated in Figure 1B, GLP-1-induced $\mathrm{NO}$ production was blocked by $\mathrm{N}^{\mathrm{G}}$-nitro- $L$-arginine methyl ester (L-NAME) (1 mmol/L), suggesting that eNOS activation mediated the effect of GLP-1 on NO release in HUVECs.

A

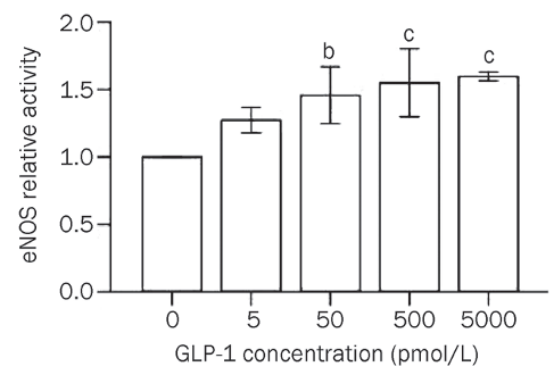

B

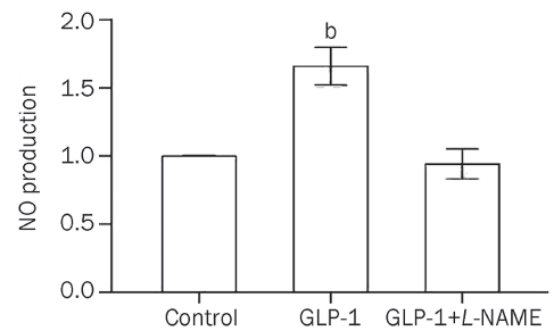

Figure 1. GLP-1 promotes endothelial nitric oxide synthase (eNOS) activity in human umbilical vein endothelial cells (HUVECs). HUVECs were incubated for $30 \mathrm{~min}$ in the absence or presence of GLP-1 (5-5000 pmol/L). Nitric oxide production was assayed using the fluorescent probe DAF-FM in the presence or absence of L-NAME $(1 \mathrm{mmol} / \mathrm{L})$. The experiment was repeated 3 times, and the values are means $\pm S D$. ${ }^{\mathrm{b}} P<0.05,{ }^{\mathrm{c}} P<0.01$ compared with control.

\section{GLP-1 stimulates the phosphorylation of eNOS in HUVECs}

The activity of eNOS is regulated by phosphorylation at multiple sites. The most thoroughly studied site is the activation site ser-1177 ${ }^{[17]}$. Several protein kinases, including Akt/PKB, PKA, and AMPK, activate eNOS by phosphorylating ser-1177 in response to various stimuli, such as insulin and fluid shear stress $^{[18,19]}$. We thus investigated whether GLP-1 increases ser-1177 phosphorylation. The incubation of HUVECs with GLP-1 (5000 pmol/L) for 5, 10, and $30 \mathrm{~min}$ increased the phosphorylation of eNOS (ser-1177), with the most significant effect being obtained at 5 min (Figure 2A). For the dose course study, 500-5000 but not 50 pmol/L GLP-1 significantly enhanced the phosphorylation of eNOS at ser-1177 (Figure 2B).

\section{The effects of GLP-1 on eNOS MRNA and protein levels in HUVECS}

Cells were incubated with 5000 pmol/L GLP-1 for 6, 12, 24, and $48 \mathrm{~h}$. Real-time RT-PCR revealed no significant difference in eNOS mRNA expression between these groups (Figure 2C). Western blot analysis indicated that GLP-1 could upregulate eNOS protein expression after a 48-h incubation (Figure 2D).

\section{The GLP-1R and GLP-1(9-36)-related pathways are involved in} the effect of GLP-1 on eNOS

Exenatide is a synthesized GLP-1 receptor agonist, and it may mimic GLP-1R-mediated effects, such as GLP-1-stimulated insulin release. To determine whether a GLP-1R-dependent or GLP-1(9-36)-related pathway (ie, GLP-1R independent) is responsible for the effects of GLP-1 on eNOS, we investigated the influence of exenatide and GLP-1(9-36) on eNOS in HUVECs. We also confirmed the expression of GLP-1R and DPP-4 proteins in HUVECs ${ }^{[15,20]}$ by Western blot analysis (Figure 3A). Like GLP-1, exenatide or GLP-1(9-36) treatment (5000 pmol/L) effectively promoted eNOS activity (Figure $3 \mathrm{~B})$, the phosphorylation of eNOS at ser-1177 (Figure 3C) and eNOS protein levels (Figure 3D) in HUVECs. Exenatide and GLP-1 had no effect on eNOS mRNA expression, but GLP-1(936) increased the mRNA levels approximately twofold after an incubation period of $48 \mathrm{~h}$ (Figure 3E). To further confirm the involvement of the GLP-1R-dependent and GLP-1(9-36)related pathways in the effects of GLP-1 on eNOS, we investigated the influence of GLP-1R blockage and DPP-4 inhibition on the effects of GLP-1. The synthesized GLP-1R antagonist exendin(9-39) and the DPP-4 inhibitor sitagliptin, which can abolish GLP-1(9-36) formation, were employed in this study. The incubation of $5000 \mathrm{pmol} / \mathrm{L}$ exendin(9-39) or sitagliptin (Figure 3F-3H) partially blocked the GLP-1-induced enhancement of eNOS activity, phosphorylation and total eNOS protein levels. Unexpectedly, cotreatment with exendin(9-39) and sitagliptin, compared with each single treatment, further attenuated but could not completely abolish the aforementioned upregulatory effects of GLP-1 on eNOS (Figure 3F-3H).

\section{Discussion}

Because GLP-1 induces endothelium-dependent vasodilation in human and rat arteries, we speculate that it may activate eNOS and increase NO production. eNOS plays an important role in maintaining normal endothelial function, and its dysfunction may be significantly associated with the cardiovascular complications in T2DM. Here, we investigated the effects of GLP-1 on the activation and expression of eNOS in HUVECs and whether GLP-1R and GLP-1(9-36) mediate these effects. Administration of 500-5000 pmol/L of GLP-1 promoted eNOS activity and phosphorylation at ser-1177 after 5 
A
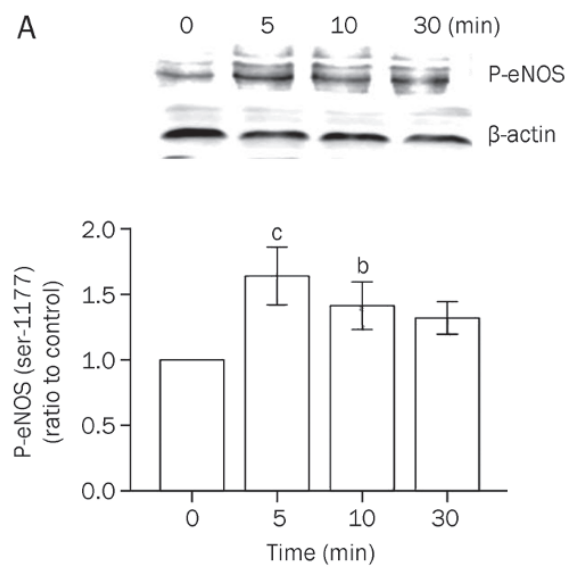

C

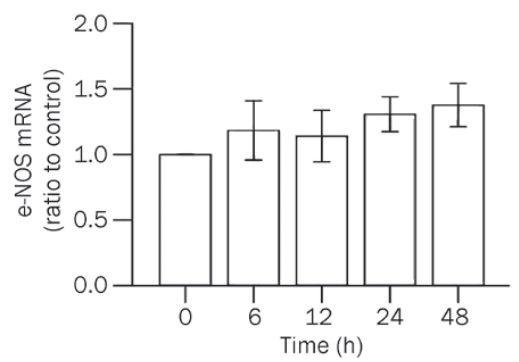

B
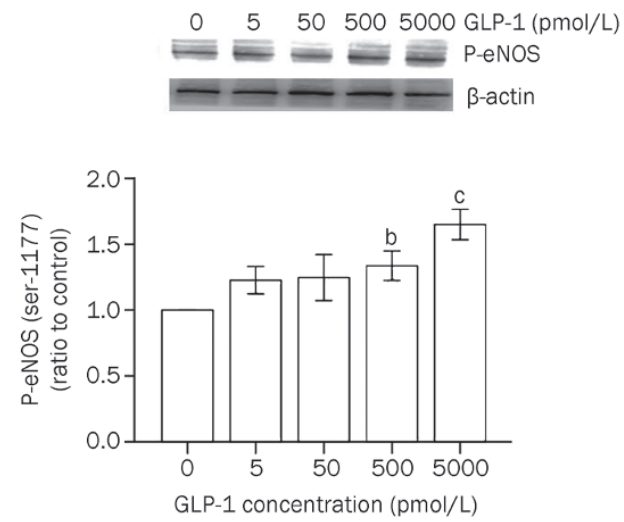

D
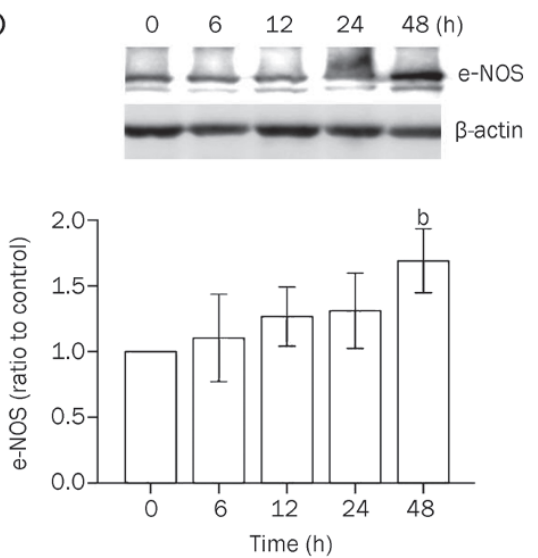

Figure 2. Effects of GLP-1 on eNOS Ser-1177 phosphorylation, mRNA and protein expression in HUVECs. (A) Cells were incubated in the presence of GLP-1 (5000 pmol/L) for 0 (control), 5, 10, and 30 min to investigate the time course of the effect on eNOS phosphorylation. (B) Cells were incubated in the absence (control) or presence of GLP-1 (5-5000 pmol/L) for 5 min to investigate the dose-dependence of the effect. eNOS phosphorylated at ser-1177 (P-eNOS 1177) was examined by Western blot analysis. Representative experiments are both shown in the upper parts. Band intensities, after normalization to $\beta$-actin, are expressed as ratios of control. Mean \pm SD. $n=4$. ${ }^{b} P<0.05,{ }^{c} P<0.01$ compared with control. (C and $D$ ) Before being harvested, cells underwent 0- (as control), 6-, 12-, 24-, and 48-h exposure to GLP-1 (5000 pmol/L). (C) eNOS mRNA levels, determined by real-time RT$P C R$ and normalized to $\beta$-actin mRNA, were expressed as mean \pm SD. (D) Total eNOS protein levels, determined by Western blot analysis and normalized to $\beta$-actin protein, are expressed as mean \pm SD. Representative experiment is shown in the upper part. $n=4$. ${ }^{b} P<0.05$ compared with control.

min in HUVECs. After a 48-h incubation, GLP-1 elevated total eNOS protein but had no effect on eNOS mRNA levels. Both exenatide and GLP-1(9-36) increased eNOS activity, phosphorylation and total protein expression. In addition, GLP-1(9-36) elevated eNOS mRNA levels. Exendin(9-39) and sitagliptin, alone or in combination, partially blocked the GLP-1-induced enhancement of eNOS activity, phosphorylation and total protein levels. Taken together, our results suggest that GLP-1 upregulates eNOS activity and protein expression through the GLP-1R-dependent and GLP-1(9-36)-related pathways in HUVECs.

The concentration of 5-5000 pmol/L covers the physiological and pharmacological concentrations of GLP-1, thus this study seeks to observe the effects of GLP-1 within this range of concentration. Figure 1 shows that 50-5000 pmol/L of GLP-1 can increase the concentration of NO. In addition, we observed the effect of GLP-1 on eNOS phosphorylation,
mRNA and protein expression (Figures 2 and 3). The observation of these three changes needs to explore the right time point. Taking into account that the relative high concentration of treatment factors may more easily produce the effects, we thus selected the $5000 \mathrm{pmol} / \mathrm{L}$ concentration for the experiment. After clarifying that this concentration could enhance eNOS phosphorylation and protein levels and determining the appropriate time point, we further observed the effect of other concentrations of GLP-1. Figure 2B shows that within 5-5000 pmol/L range, only 500-5000 pmol/L of GLP-1 can cause elevated levels of phosphorylation. However, a lower concentration (5-500 pmol/L) of GLP-1 does not cause changes in protein levels (data not shown), suggesting that only high concentrations of GLP-1 can result in eNOS expression upregulation. As indicated in Figure 1, $5000 \mathrm{pmol} / \mathrm{L}$ of GLP-1 did not cause eNOS mRNA change in the time period we observed, thus we did not further observe the effect of other concentrations. 
A

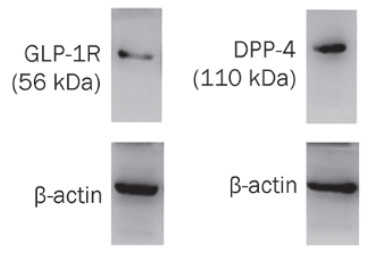

D

Control GLP-1 (9-36) Exenatide
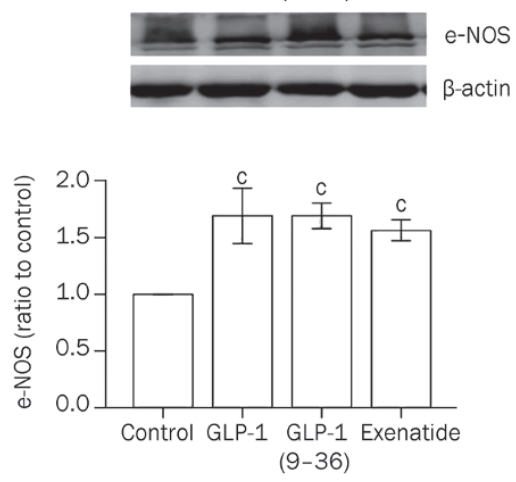

G

Control G G+E G+S G+E+S

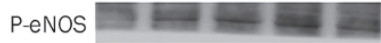

$\beta$-actin -

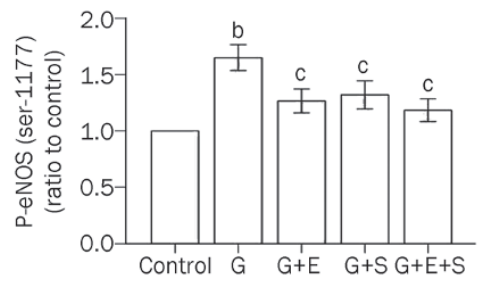

B

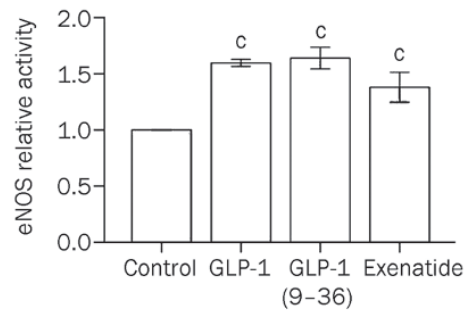

E

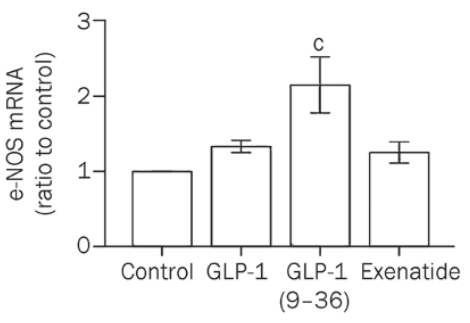

$\mathrm{H}$

Control G G+E G+S G+E+S e-NOS $=$ $\beta$-actin

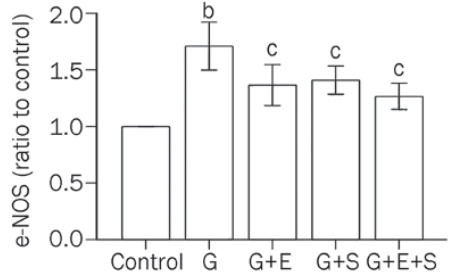

C

GLP-1

Control GLP-1 (9-36) Exenatide
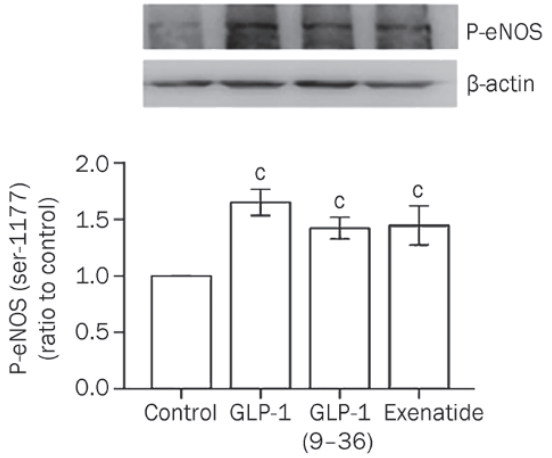

$\mathrm{F}$

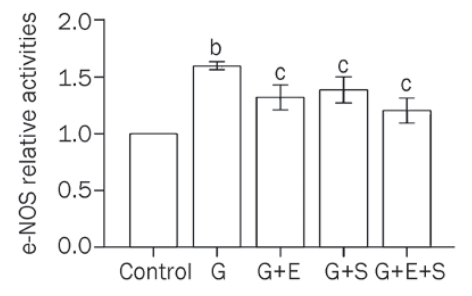

Figure 3. GLP-1R-dependent pathway and GLP-1(9-36)-related pathway are involved in effects of GLP-1 on eNOS levels in HUVECs. (A) GLP-1R and DPP-4 proteins were detected in HUVECs by Western blot analysis. Cells were incubated in the presence or absence (control) of GLP-1, GLP-1(9-36) or exenatide (GLP-1R agonist) (all at 5000 pmol/L) for the following times (B, C, D, E). Cells were incubated with GLP-1 in the presence of exendin (9-39) $(\mathrm{G}+\mathrm{E})$ or sitaglipin $(\mathrm{G}+\mathrm{S})$ or both $(\mathrm{G}+\mathrm{E}+\mathrm{S})$ for the following times $(\mathrm{F}, \mathrm{G}, \mathrm{H})$. (B, F) After 30-min incubation, eNOS activity was determined by NO content in cells. (C, G) After 5-min incubation, phosphorylation of eNOS at ser-1177 was examined by Western blot analysis. (D, H) After 48-h incubation, total eNOS protein level was measured by Western blot analysis. The upper parts of C, D, G, and H show representative experiments. Data are mean $\pm S D$ after normalization to $\beta$-actin level. (E) After 48-h incubation, eNOS mRNA level was quantified by real-time RT-PCR. Data are mean $\pm S D$ after normalization to $\beta$-actin level. ${ }^{\mathrm{b}} \mathrm{P}<0.05,{ }^{\mathrm{c}} \mathrm{P}<0.01$ compared to control.

Previous research has suggested that a 30-s infusion of GLP-1 can induce pulmonary vasodilation in isolated rat lungs that can be blocked by L-NAME, an eNOS inhibitor ${ }^{[9]}$. Thus, GLP-1 may increase NO release by directly increasing eNOS activity rather than protein levels in a short time. In this study, to understand the effect of GLP-1 on eNOS activity, we observed intracellular NO levels in HUVECs incubated with
GLP-1 for $30 \mathrm{~min}$ and found that GLP-1 (50-5000 pmol/L) could increase eNOS activity. In normal individuals, the concentration of GLP-1 during the fasting state is $5-10 \mathrm{pmol} / \mathrm{L}$ and can increase to $20-40 \mathrm{pmol} / \mathrm{L}$ after meals whereas pharmacological concentrations of GLP-1 can be more than 100 $\mathrm{pmol} / \mathrm{L}^{[21,22]}$. Thus, GLP-1 may increase eNOS activity at both physiological and pharmacological concentrations. Among 
the rapid regulatory mechanisms of eNOS activity, eNOS ser1177 phosphorylation is the most important pathway and is most frequently investigated. In the present study, GLP-1induced eNOS ser-1177 phosphorylation was time dependent, $i e$, phosphorylated eNOS levels increased rapidly in a few minutes and then decreased within a short amount of time. Thus, GLP-1-induced eNOS activation may be restricted by negative feedback mechanisms, thereby limiting NO synthesis within an appropriate range to avoid adverse effects of excessive NO in endothelium.

GLP-1-induced NO synthesis and release via the activation of eNOS can explain the direct vasodilation by GLP-1. Moreover, GLP-1 can facilitate the effects of other vasodilator stimuli, such as fluid shear stress and acetylcholine ${ }^{[9-14]}$. However, in Daul salt-sensitive rats, after continuous infusion of GLP-1 for $14 \mathrm{~d}$, the vasorelaxing reaction of the isolated thoracic aorta ring in response to acetylcholine was twice that in non-GLP-1infused rats ${ }^{[12]}$, indicating that GLP-1 has a long-term effect on eNOS, especially at the mRNA and protein levels. Here, our results reveal that a 48 -h incubation of GLP-1 increases eNOS protein but not mRNA levels in HUVECs. Thus, GLP-1 regulates eNOS expression at the translational level and has no effects on gene transcription or mRNA stability.

GLP-1R mediates the main effect of GLP-1 on its target tissues, such as pancreatic islets, and plays an important role in maintaining glucose homeostasis. Indeed, GLP-1R is also expressed in endothelial cells ${ }^{[11,13,15]}$; thus, we hypothesize that the GLP-1R-dependent pathway may be involved in the endothelial effects of GLP-1. On the other hand, evidence indicates that an unknown signal pathway, which is independent of GLP-1R but is related to GLP-1(9-36), may exist and mediate certain biological effects of GLP-1. The half-life of GLP-1 in vivo is very short (only several minutes), and the cycling GLP-1 is usually present in the form of GLP-1(9-36) ${ }^{[23]}$. GLP-1(9-36) has a weak affinity with GLP-1R but cannot activate GLP-1R. Thus, it was considered either to have no biological activity or to antagonize GLP-1R to some extent ${ }^{[24]}$. Recent studies have found that GLP-1 can protect myocardium and relax blood vessels in mutagenic mice without GLP-1R. GLP-1(9-36) has a similar effect in normal and GLP-1R ${ }^{-/-}$mice. DPP-4 inhibitors, which block the conversion of GLP-1 to GLP-1(9-36), can weaken the above effects of GLP-1 ${ }^{[11]}$. Studies have also found that GLP-1(9-36) has a more intense relaxing effect on isolated mouse femoral artery rings compared with GLP-1 and that single GLP-1R agonists have no relaxing effect ${ }^{[10,11]}$. Together, these studies indicate that a GLP-1(9-36)-related, non-GLP$1 R$-dependent signaling pathway may mediate the biological effect of GLP-1. However, whether a GLP-1R-dependent or GLP-1(9-36)-related pathway mediates the effects of GLP-1 on eNOS was unknown. We thus treated HUVECS with GLP-1, exenatide (a GLP-1R agonist) and GLP-1(9-36) at the same concentrations and found that all of them could increase the activity and phosphorylation of eNOS as well as the total eNOS protein levels. In addition, like GLP-1, exenatide had no effect on eNOS mRNA levels, but GLP-1(9-36) could increase eNOS mRNA expression after a 48-h incubation. Thus, both the GLP-1R-dependent and GLP-1(9-36)-related pathways may be involved in the effect of GLP-1 on eNOS in HUVECs. Moreover, both the GLP-1R antagonist and the DPP-4 inhibitor that abolishes GLP-1(9-36) formation partially suppressed the GLP-1-induced eNOS activation, ser-1177 phosphorylation and upregulation of eNOS protein levels, further confirming the involvement of these two signaling pathways.

GLP-1 can upregulate eNOS in HUVECs, implying that, as a physiological hormone, it is involved in maintaining normal endothelial function. Thus, decreased GLP-1 levels may be a mechanism underlying the endothelial dysfunction in T2DM patients. Previous studies have demonstrated that GLP-1 and GLP-1(9-36) can lead to the vasodilation of femoral and mesenteric arteries in rats, but the GLP-1R agonist exendin- 4 and exenatide has no such effects ${ }^{[12,13]}$. This observation can be explained by the lack of GLP-1R expression in mesenteric artery intima $^{[13]}$. Thus, the increased GLP-1(9-36) may be necessary to improve the pathophysiological changes caused by the decreased secretion of GLP-1 in T2DM patients, especially in tissues without GLP-1R expression. To date, there are few studies on the GLP-1(9-36)-related signaling pathway. Further research can provide a better understanding of the physiological functions and pharmacological actions of GLP-1(9-36).

In summary, GLP-1 can increase eNOS activity and protein levels in HUVECs via both the GLP-1R-dependent and GLP-1(9-36)-related pathways. New antidiabetic drugs, including GLP-1 analogues, GLP-1R agonists and DPP-4 inhibitors, improve glucose control in T2DM patients and have direct cardiovascular-protective effects. In addition, close attention should also be paid to the difference between the cardiovascular effects of GLP-1R agonists, GLP-1 analogues and DPP-4 inhibitors.

\section{Acknowledgements}

We thank Mu-li ZHAO for her assistance in cell culture. We are grateful to editors at BiomedWorld Bioscience Limited for their assistance in preparing this article.

\section{Author contribution}

Li DING and Jin ZHANG designed and performed the research and analyzed the data; and Li DING wrote the paper.

\section{References}

1 Yang Z, Ming XF. Recent advances in understanding endothelial dysfunction in atherosclerosis. Clin Med Res 2006; 4: 53-65.

2 Bauer V, Sotnikova R. Nitric oxide - the endothelium-derived relaxing factor and its role in endothelial functions. Gen Physiol Biophys 2010; 29: 319-40.

3 Stankevicius E, Kevelaitis E, Vainorius E, Simonsen U. Role of nitric oxide and other endothelium-derived factors. Medicina (Kaunas) 2003; 39: 333-41.

4 Drucker DJ. The biology of incretin hormones. Cell Metab 2006; 3: 153-65.

5 Drucker DJ, Nauck MA. The incretin system: glucagon-like peptide-1 receptor agonists and dipeptidyl peptidase-4 inhibitors in type 2 diabetes. Lancet 2006; 368: 1696-705.

6 Meier JJ, Nauck MA. Incretins and the development of type 2 
diabetes. Curr Diab Rep 2006; 6: 194-201.

7 Toft-Nielsen MB, Damholt MB, Madsbad S, Hilsted LM, Hughes TE, Michelsen BK, et al. Determinants of the impaired secretion of glucagon-like peptide-1 in type 2 diabetic patients. J Clin Endocrinol Metab 2001; 86: 3717-23.

8 Briones M, Bajaj M. Exenatide: a GLP-1 receptor agonist as novel therapy for Type 2 diabetes mellitus. Expert Opin Pharmacother 2006; 7: 1055-64.

9 Golpon HA, Puechner A, Welte T, Wichert PV, Feddersen CO. Vasorelaxant effect of glucagon-like peptide-(7-36)amide and amylin on the pulmonary circulation of the rat. Regul Pept 2001; 102: 81-6.

10 Nathanson D, Erdogdu O, Pernow J, Zhang Q, Nystrom T. Endothelial dysfunction induced by triglycerides is not restored by exenatide in rat conduit arteries ex vivo. Regul Pept 2009; 157: 8-13.

11 Ban K, Noyan-Ashraf MH, Hoefer J, Bolz SS, Drucker DJ, Husain M. Cardioprotective and vasodilatory actions of glucagon-like peptide 1 receptor are mediated through both glucagon-like peptide 1 receptordependent and -independent pathways. Circulation 2008; 117 : 2340-50.

12 Yu M, Moreno C, Hoagland KM, Dahly A, Ditter K, Mistry M, et al. Antihypertensive effect of glucagon-like peptide 1 in Dahl saltsensitive rats. J Hypertens 2003; 21: 1125-35.

13 Nystrom T, Gutniak MK, Zhang Q, Zhang F, Holst JJ, Ahren B, et al. Effects of glucagon-like peptide-1 on endothelial function in type 2 diabetes patients with stable coronary artery disease. Am J Physiol Endocrinol Metab 2004; 287: E1209-15.

14 Basu A, Charkoudian N, Schrage W, Rizza RA, Basu R, Joyner MJ. Beneficial effects of GLP-1 on endothelial function in humans: dampening by glyburide but not by glimepiride. Am J Physiol Endocrinol Metab 2007; 293: E1289-95.

15 Ishibashi Y, Matsui T, Takeuchi M, Yamagishi S. Glucagon-like peptide-1 (GLP-1) inhibits advanced glycation end product (AGE)induced up-regulation of VCAM-1 mRNA levels in endothelial cells by suppressing AGE receptor (RAGE) expression. Biochem Biophys Res Commun 2010; 391: 1405-8.
16 Ban K, Kim KH, Cho CK, Sauve M, Diamandis EP, Backx PH, et al. Glucagon-like peptide (GLP)-1(9-36)amide-mediated cytoprotection is blocked by exendin(9-39) yet does not require the known GLP-1 receptor. Endocrinology 2010; 151: 1520-31.

17 Chen ZP, Mitchelhill KI, Michell BJ, Stapleton D, Rodriguez-Crespo I, Witters LA, et al. AMP-activated protein kinase phosphorylation of endothelial NO synthase. FEBS Lett 1999; 443: 285-9.

18 Dimmeler S, Fleming I, Fisslthaler B, Hermann C, Busse R, Zeiher AM. Activation of nitric oxide synthase in endothelial cells by Aktdependent phosphorylation. Nature 1999; 399: 601-5.

19 Fulton D, Gratton JP, McCabe TJ, Fontana J, Fujio Y, Walsh K, et al. Regulation of endothelium-derived nitric oxide production by the protein kinase Akt. Nature 1999; 399: 597-601.

20 Liu H, Hu Y, Simpson RW, Dear AE. Glucagon-like peptide-1 attenuates tumour necrosis factor-alpha-mediated induction of plasminogen [corrected] activator inhibitor-1 expression. J Endocrinol 2008; 196: 57-65.

21 Hojberg PV, Vilsboll T, Rabol R, Knop FK, Bache M, Krarup T, et al. Four weeks of near-normalisation of blood glucose improves the insulin response to glucagon-like peptide-1 and glucose-dependent insulinotropic polypeptide in patients with type 2 diabetes. Diabetologia 2009; 52: 199-207.

22 Vilsboll T, Krarup T, Madsbad S, Holst JJ. Both GLP-1 and GIP are insulinotropic at basal and postprandial glucose levels and contribute nearly equally to the incretin effect of a meal in healthy subjects. Regul Pept 2003; 114: 115-21.

23 Mentlein R. Mechanisms underlying the rapid degradation and elimination of the incretin hormones GLP-1 and GIP. Best Pract Res Clin Endocrinol Metab 2009; 23: 443-52.

24 Owji AA, Khoshdel Z, Sanea F, Panjehshahin MR, Shojaee Fard $\mathrm{M}$, Smith DM, et al. Effects of intracerebroventricular injection of glucagon like peptide-1 and its related peptides on serotonin metabolism and on levels of amino acids in rat hypothalamus. Brain Res 2002; 929: 70-5. 